\title{
Toothbrushing link to seizures
}

Toothbrushing may trigger seizures in certain people with epilepsy according to an article published in Neurology (2007; 68: 769771), the journal of the American Academy of Neurology.

Researchers say lesions in a specific part of the brain may be a cause in some people and the article reviewed the cases of three adults with epilepsy who experienced seizures while brushing their teeth. Two of the adults reported that some of their seizures occurred when they brushed certain areas of their mouth. The seizures varied from jerking and twitching of the face to salivating vigorously. One patient was unable to let go of the toothbrush during the seizure. The seizures were confirmed by video monitoring.

Using an MRI, researchers found all three patients had lesions in the somatosensory area of the brain, which is close to the hand and speech motor areas. 'The rhythmic act of brushing teeth may excite an already overly excitable area of the brain and is similar to photosensitive epilepsy, which involves seizures triggered by flashing lights and moving patterns,' said study author Wendyl D'Souza, who is with the Department of Neurology and Neurological Research at St Vincent's Hospital, the University of Melbourne, Australia.

She added that since toothbrushing involved persistent rhythmic action, this may explain why this trigger is more likely to induce seizures in the somatosensory area of the brain compared to other oral stimuli, such as eating.

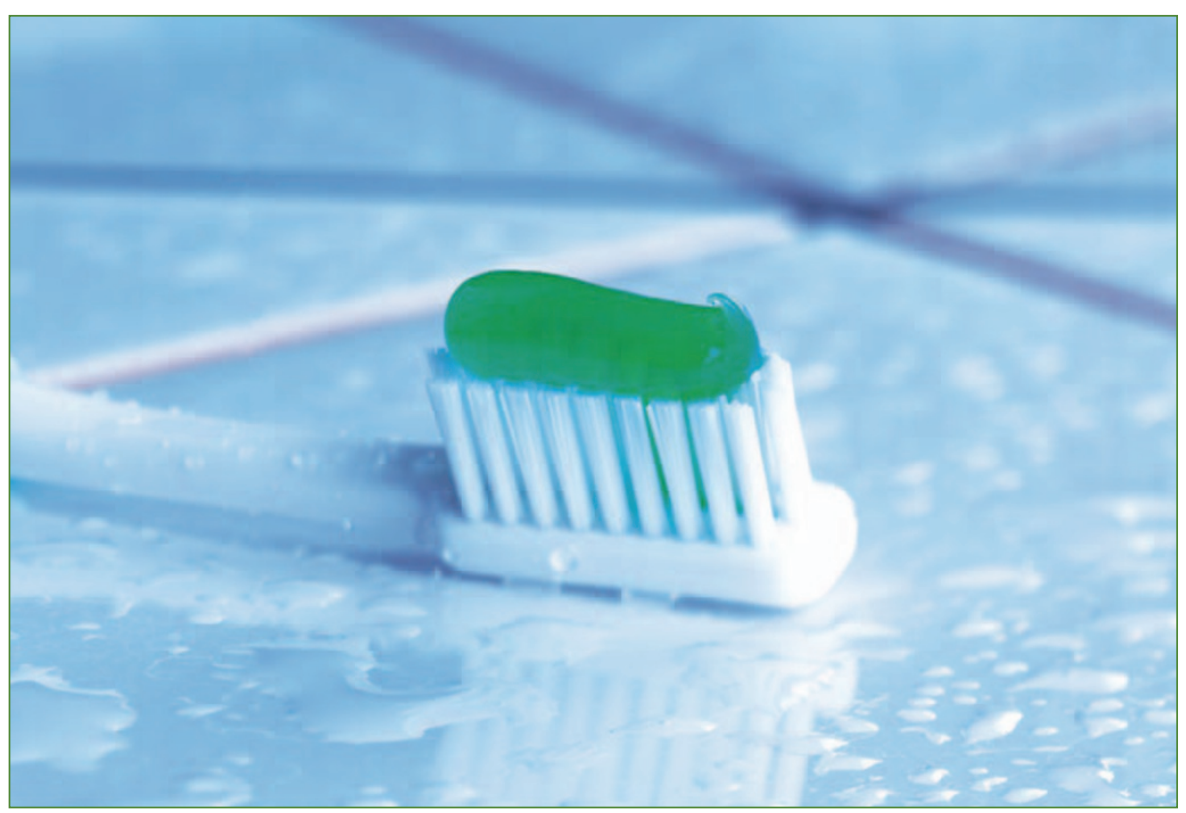

\section{DCP registration: one year on}

It has been just under a year since the GDC opened the dental care professional (DCP) register. Registration for dental nurses and technicians will become compulsory on 31 July 2008.

The GDC is encouraging DCPs to join the register during the two-year transitional period, which will enable dental nurses and technicians who have been working in their role for a number of years to join the register on the basis of validated experience. Dental nurses and technicians joining the register after 30 July 2008 will need to have a GDCrecognised qualification.

The process has not been without its challenges. Some DCPs paid for medical examinations as part of the application process, but the GDC subsequently changed its guidance in this area, resulting in refunds for those who had paid for them.

GDC Chief Executive \& Registrar, Duncan Rudkin said, 'Changes were made in recog- nition of the fact that some roles were more exposure-prone than others and therefore carry different degrees of risk for patients.'

The overall response to DCP registration has been positive with the GDC sending out approximately 5,000 registration application packs in the first month in response to requests from DCPs keen to be among the first new registrants. Currently just over 3,000 DCPs have registered, including 2,784 dental nurses, 273 dental technicians and 12 clinical dental technicians.

He adds, 'When you register with us, we give you a licence to work as a dental professional in the UK. From 31 July 2008, you won't be able to work as a dental nurse or dental technician without this licence.'

Duncan continues, 'If you have already received a pack from us please complete and return your application as soon as possible; it currently takes just under eight weeks to process your application. Applying now will take away any worries about having to register at the last minute and running the risk of being unregistered. This is especially important if you want to apply on the basis of your experience because after 30 July 2008 the only way on to the register will be with a qualification - so make sure you don't miss the boat.'

The GDC also plans to introduce a continuing professional development (CPD) scheme for DCPs in July 2008. He says, 'As a registered DCP you will need to keep upto-date in your work through continuing professional development. In 2008 we plan to introduce compulsory CPD requirements which will include core subjects you'll need to keep updated on. We will tell you more about this over the coming year.'

For more information about registering contact the GDC on 02078873800 , by email at gdcregistration@gdc-uk.org or visit www.gdc-uk.org. 